\title{
A Swan Song for CCSVI
}

\author{
Jim Reekers
}

Received: 12 November 2013/Accepted: 6 December 2013/Published online: 9 January 2014

(C) Springer Science+Business Media New York and the Cardiovascular and Interventional Radiological Society of Europe (CIRSE) 2014

\section{Introduction}

The introduction of the medical entity termed CCSVI, an appealing abbreviation for the narrowing of the jugular vein, and its suggested relationship to multiple sclerosis (MS) has caused many controversies among interventional radiologists worldwide. CVIR has produced an early and balanced statement pointing out the lack of evidence, along with a warning not to start the treatment of balloon dilatation of the jugular vein [1]. Others have been less cautious, focusing instead more on the perceived opportunities of this new technique. Now that two major studies $[2,3]$ have shown that CCSVI does not exist-not in MS and not in any other group, such as siblings of MS patients and healthy volunteers-it is perhaps time to draw some conclusions regarding the introduction of future new and socalled promising interventional procedures.

\section{CCSVI}

On the basis of the finding that some early MS lesions are located around a vein, Zamboni hypothesized that venous congestion plays an important role in MS. In his first paper in 2006, he called this "the big idea" [4]. Not many people picked up on this idea at first. However, this changed dramatically when Zamboni and coworkers laid down five criteria for ultrasound color Doppler imaging for venous

J. Reekers $(\bowtie)$

Department of Radiology, AMC, University of Amsterdam,

Meibergdreef 9, 1105 AZ Amsterdam, The Netherlands

e-mail: j.a.reekers@amc.uva.nl abnormalities related to MS [5, 6]. They found a high percentage of MS patients with at least two of the so-called Zamboni criteria, while this was not found in healthy volunteers $[5,6]$. They called the pathological condition based on this criteria chronic cerebrospinal venous insufficiency, or CCSVI. Despite large-scale skepticism, the theory found many followers among interventionists who denied the growing evidence that CCSVI did not exist and that the diagnostic criteria were flawed and open to multiple interpretations and bias [7]. To date, Zamboni and coworkers have produce a pile of circumstantial "evidence" to support their hypotheses of CCSVI and MS. However, the only evidence that would really be of importance - a blinded randomized controlled trial with a sham arm - was not among this evidence.

There are now two recent publications of good and unbiased quality, studying approximately 2000 patients, that show that CCSVI has no higher prevalence in MS than in any other group of patients without MS or healthy volunteers, which proves the hypothesis of CCSVI and venous congestion in relation to MS to be wrong [2,3]. Next to the five Zamboni criteria, many followers were encouraged by the observation that some MS patients responded positively to the balloon dilatation treatment. MS is still a not very well-understood disease, but it has been well established that psychology and the placebo effect also play important roles in the well-being of MS patients. That is why many other MS treatments, such as stem cell injections, herbal tea, and biofeedback, to mention only a few, sometimes seem to work. This placebo effect, however, is never lasting, and on the Internet may be found many testimonies by disappointed MS patients. After the publication of these high-quality data, CCSVI has been scientifically proven to not exist, and therefore, there is no evidence to offer balloon dilatation treatment to patients with MS. 


\section{What Are the Lessons to Be Learned?}

Any new hypothesis or treatment in medicine should have a solid, logical, and explainable biophysiological basis. Any diagnostic test in relation to a new hypothesis and/or treatment should be evaluated for flaws and bias before it is ever applied.

At issue are the following: Is a test operator dependent? Was a gold standard used? Was the cutoff for sensitivity and specificity appropriate? What are the positive and negative likelihood ratios? Is the test appropriate for a specific population? But many more criteria need to be assessed, such as selection bias and sample size [8]. None of these was considered for the Zamboni criteria for ultrasound color Doppler imaging.

Single-center experiences can be the start of a new intervention, but independent confirmation by at least one other independent source is mandatory. Lack of randomized data years after the introduction of a new medical treatment is unacceptable. Widespread acceptance of a new technique before good scientific evidence is available is dangerous, and it also makes any randomized trial very difficult: the initiator of such trials is often accused of unethical behavior by withholding a "proven" treatment to patients who need it. Advertisement of new and unproven medical treatments through the media and the Internet can potentially result in compromised patient safety. A fast accumulation of single-center publications, which are then used as a reference source for new publications from the same groups, bears resemblance to a Ponzi scheme.

Are we going to be more alert in the future-not to be too enthusiastic about a new but still unproven treatment in interventional radiology? I hope we will. However, the need of many physicians not to withhold from their patients what seems the best treatment available is understandable. However, absence of evidence is not the same as evidence of absence.

Health care providers never considered reimbursement for the "liberation treatment" — not even in Canada, were the media pressure was high. Currently the absence of evidence is the same as the absence of reimbursement. Therefore, we should also try to introduce any new interventional radiological techniques along the lines of evidence-based medicine to guarantee proper reimbursement for our patients.

Balloon angioplasty and stenting of the jugular vein for CCSVI should have been stopped 4 years ago as a result of insufficient evidence. This would have prevented false hope from gaining ground for MS patients and their physicians.

Conflict of interest The author declares that he has no conflict of interest.

\section{References}

1. Reekers JA, Lee MJ, Belli AM, Barkhof F (2011) Cardiovascular and Interventional Radiological Society of Europe commentary on the treatment of chronic cerebrospinal venous insufficiency. Cardiovasc Interv Radiol 34:1-2

2. Comi G, Battaglia MA, Bertolotto A et al (2013) Italian multicentre observational study of the prevalence of CCSVI in multiple sclerosis (CoSMo study): rationale, design, and methodology. Neurol Sci 34:1297-1307

3. Traboulsee AL, Knox KB, Machan L et al (2013) Prevalence of extracranial venous narrowing on catheter venography in people with multiple sclerosis, their siblings, and unrelated healthy controls: a blinded, case-control study. Lancet. doi:10.1016/ S0140-6736(13)61747-X

4. Zamboni $P$ (2006) The big idea: iron-dependent inflammation in venous disease and proposed parallels in multiple sclerosis. J R Soc Med 99:589-593

5. Zamboni P, Galeotti R, Menegatti E et al (2009) Chronic cerebrospinal venous insufficiency in patients with multiple sclerosis. J Neurol Neurosurg Psychiatry 80:392-399

6. Zamboni P, Menegatti E, Gaeleotti R et al (2009) The value of cerebral Doppler venous heamodynamics in the assessment of multiple sclerosis. J Neurol Sci 282:21-27

7. Valdueza JM, Doepp F, Schreiber SJ et al (2013) What went wrong? The flawed concept of cerebrospinal insufficiency. J Cereb Blood Flow Metab 33:657-668

8. Knottnerus JA, van Weel C, Muris JW (2002) Evaluation of diagnostic procedures. BMJ 324(7335):477-780 erratum BMJ 2002;324(7350):1391 